BULLETIN (New Series) OF THE

AMERICAN MATHEMATICAL SOCIETY

Volume 49, Number 1, January 2012, Pages 73-90

S 0273-0979(2011)01360-X

Article electronically published on October 19, 2011

\title{
SPACES OF GRAPHS AND SURFACES: ON THE WORK OF SØREN GALATIUS
}

\author{
ULRIKE TILLMANN
}

\begin{abstract}
We put Soren Galatius's result on the homology of the automorphism group of free groups into context. In particular we explain its relation to the Mumford conjecture and the main ideas of the proofs.
\end{abstract}

\section{INTRODUCTION}

Galatius's most striking result is easy enough to state. Let $\Sigma_{n}$ be the symmetric group on $n$ letters, and let $F_{n}$ be the free (non-abelian) group with $n$ generators. The symmetric group $\Sigma_{n}$ acts naturally by permutation on the $n$ generators of $F_{n}$, and every permutation thus gives rise to an automorphism of the free group. Galatius proves that the map $\Sigma_{n} \hookrightarrow \operatorname{Aut} F_{n}$ in homology induces an isomorphism in degrees less than $(n-1) / 2$ and in rational homology in degrees less than $2 n / 3$. The homology of the symmetric groups in these ranges is well understood. In particular, in common with all finite groups, it has no non-trivial rational homology. By Galatius's theorem, in low degrees this is then also true for $\operatorname{Aut} F_{n}$ :

$$
H_{*}\left(\operatorname{Aut} F_{n}\right) \otimes \mathbb{Q}=0 \quad \text { for } \quad 0<*<2 n / 3,
$$

as had been conjectured by Hatcher and Vogtmann.

We will put this result in context and explain the connection with previous work on the mapping class group of surfaces and the homotopy theoretic approach to a conjecture by Mumford in [37] on its rational, stable cohomology. Galatius's proof in [14] is inspired by this and at the same time improves the methods significantly. This in turn has led to further deep insights into the topology of moduli spaces of manifolds also in higher dimensions.

Section 2 will introduce the more algebraic concepts, and in section 3 we will take a more geometric point of view. The latter leads naturally into section 4 where the main ideas behind the proof, which is geometric in nature, are explained. Section 5 contains further results and directions.

\section{Groups And Their (CO)homology}

We will first step back and say a bit more about the groups mentioned above and discuss their (co)homology in essentially algebraic terms. There are many parallels between mapping class groups and automorphisms of free groups. Indeed, much of the work on $\operatorname{Aut} F_{n}$ has been inspired by the work on the mapping class group as these groups show very similar behavior.

Received by the editors July, 18, 2011, and, in revised form, August 7, 2011.

2010 Mathematics Subject Classification. Primary 20J06.

(C)2011 American Mathematical Society Reverts to public domain 28 years from publication 
We refer readers to [43] and the references therein for more details on the automorphisms group of free groups. A convenient reference for the mapping class group is the recent book [12. The basics on cohomology of groups used here can all be found in 5 .

2.1. Groups of primary interest. We will first introduce the discrete groups that we will mainly be interested in.

2.1.1. Symmetric groups. $\Sigma_{n}$ is the permutation group on $n$ letters. It is finite of size $n$ !, and hardly needs further introduction. It has a presentation with generators the transpositions $\sigma_{1}, \ldots, \sigma_{n-1}$ that swap two adjacent letters, and relations $\sigma_{i}^{2}=1$ for all $i$, and the braid relations $\sigma_{i} \sigma_{i+1} \sigma_{i}=\sigma_{i+1} \sigma_{i} \sigma_{i+1}$ for all $i$, and $\sigma_{i} \sigma_{j}=\sigma_{j} \sigma_{i}$ for $|i-j|>1$.

2.1.2. Automorphism groups of free groups. Let $F_{n}$ be the free (non-abelian) group generated by $n$ letters. Its group of automorphisms $\mathrm{Aut} F_{n}$ is the group of invertible homomorphisms of $F_{n}$ to itself. Closely related is the outer automorphism group Out $F_{n}$. Conjugation by a fixed element in a group defines an automorphism. The set of all such automorphisms forms a normal subgroup $\operatorname{Inn} F_{n}$ of inner automorphisms. Out $F_{n}$ is the quotient group of $\operatorname{Aut} F_{n}$ by this group.

The canonical map from the free group $F_{n}$ to the free abelian group $\mathbb{Z}^{n}$ induces a surjective homomorphism

$$
L: \operatorname{Aut} F_{n} \longrightarrow \mathrm{GL}(n, \mathbb{Z})
$$

to the general linear group. As the inner automorphisms of $\mathbb{Z}^{n}$ are trivial, we see that $L$ factors through $\operatorname{Out} F_{n}$. So both $\operatorname{Aut} F_{n}$ and $\operatorname{Out} F_{n}$ are infinite. They also both contain a copy of the symmetric group $\Sigma_{n}$ which acts naturally on the generators of $F_{n}$ and $\mathbb{Z}^{n}$ by permutation.

The inverse image under $L$ of the special linear group defines a subgroup $S$ Aut $F_{n}$ in Aut $F_{n}$ of index 2. A set of generators for this subgroup are the Nielsen transformations $\lambda_{i j}$ and $\rho_{i j}$, which multiply the $i$ th generator of $F_{n}$ by the $j$ th on the left and right, respectively, and leave all other generators fixed. A nice presentation of this subgroup is given by [18. To get a full set of generators, one needs to add an automorphism of determinant -1 such as the map that sends the first generator to its inverse and leaves all other generators fixed.

$F_{n}$ is the fundamental group of a bouquet of $n$ circles, or any graph $G_{n}$ with Euler characteristic $1-n$ more generally. Let $\operatorname{HtEq}\left(G_{n}\right)$ denote the space of homotopy equivalences of $G_{n}$, and let $\operatorname{HtEq}\left(G_{n} ; *\right)$ denote the subspace of homotopy equivalences that fix a basepoint. Their groups of components are Out $F_{n}$ and
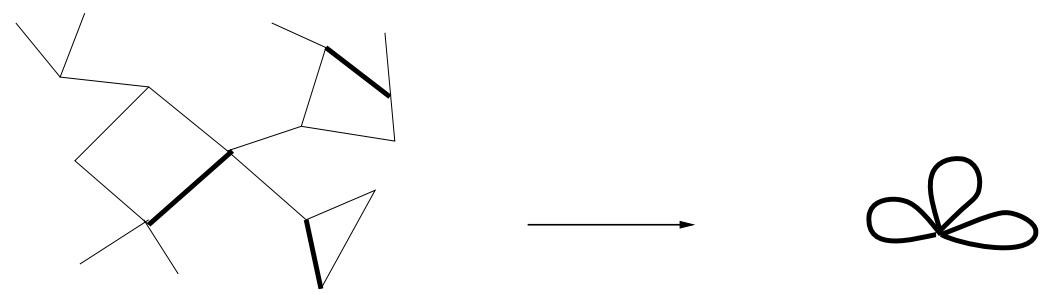

Figure 1. Collapsing a maximal tree defines a homotopy equivalence. 
Aut $F_{n}$, respectively. Furthermore, each connected component is contractible. We thus have homotopy equivalences

$$
\operatorname{HtEq}\left(G_{n} ; *\right) \simeq \operatorname{Aut} F_{n} \quad \text { and } \operatorname{HtEq}\left(G_{n}\right) \simeq \operatorname{Out} F_{n} .
$$

2.1.3. Mapping class groups of surfaces. Let $S_{g, 1}$ be an oriented surface of genus $g$ with one boundary component, and let $\operatorname{Diff}^{+}\left(S_{g, 1} ; \partial\right)$ be its group of diffeomorphisms that fix the boundary pointwise and (hence necessarily) fix any orientation of the surface. The mapping class group $\Gamma_{g, 1}$ is the group $\pi_{0}\left(\operatorname{Diff}^{+}\left(S_{g, 1} ; \partial\right)\right)$ of connected components. Similarly one defines the mapping class group $\Gamma_{g}:=$ $\pi_{0}\left(\operatorname{Diff}^{+}\left(S_{g}\right)\right)$ of an oriented, closed surface $S_{g}$ of genus $g$. It is generated by Dehn twists around simple closed curves defined by the following procedure: cut the surface along such a curve, twist one side by a full turn, and glue it back. A useful presentation was found by Wajnryb [45. It is not difficult to see that when two curves intersect, once the associated Dehn twists satisfy the braid relation, relation $a b a=b a b$; when two curves do not intersect, their associated Dehn twists commute, $a b=b a$.

Diffeomorphisms act on the first homology $H_{1}\left(S_{g}\right)=\mathbb{Z}^{2 g}$ of the underlying surface, and when they are orientation preserving, they preserve the intersection form. This defines a surjective representation

$$
\Gamma_{g} \longrightarrow \mathrm{SP}(2 g, \mathbb{Z}) \text {. }
$$

When the Euler characteristic of the underlying surfaces is negative, Earle and Eells 8 showed that the connected components of the diffeomorphism groups are contractible. Thus in this case we also have homotopy equivalences

$$
\operatorname{Diff}^{+}\left(S_{g, 1}\right) \simeq \Gamma_{g, 1} \quad \text { and } \quad \operatorname{Diff}^{+}\left(S_{g}\right) \simeq \Gamma_{g} .
$$

2.1.4. Natural homomorphisms between these groups. We have already seen that the maps

$$
\Sigma_{n} \longrightarrow \operatorname{Aut} F_{n} \longrightarrow \mathrm{Out} F_{n}
$$

define an inclusion of $\Sigma_{n}$ into $\operatorname{Aut} F_{n}$ and $\operatorname{Out} F_{n}$; here, as before, the first map is induced by the permutation action on a set of generators for $F_{n}$ and the second map is the quotient map. By gluing a disc to the boundary of the surface $S_{g, 1}$ and extending diffeomorphisms by the identity, we get a natural map

$$
\Gamma_{g, 1} \longrightarrow \Gamma_{g}
$$

which is well known to be surjective. Finally, every diffeomorphism of $S_{g, 1}$ induces an automorphism of the fundamental group $\pi_{1} S_{g, 1}=F_{2 g}$. This defines the maps

$$
\rho^{+}: \Gamma_{g, 1} \longrightarrow \text { Aut } F_{2 g} \text { and } \rho^{+}: \Gamma_{g} \longrightarrow \text { Out } F_{2 g} \text {. }
$$

Indeed, the latter is an inclusion onto an index two subgroup by a theorem due to Dehn, Nielsen, and Baer.

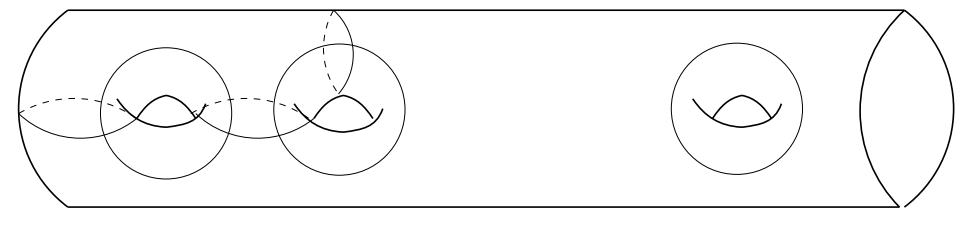

Figure 2. Dehn twist around the $2 g+1$ indicated curves generates $\Gamma_{g, 1}$. 
2.2. Group (co)homology. One way to study a discrete group $G$ is through its homology $H_{n}(G)$ and cohomology $H^{n}(G)$ groups. These groups can be defined purely algebraically or as the homology and cohomology of a space $B G$. The space $B G$ is determined (up to homotopy) by the fact that its fundamental group is $G$ and its universal cover is contractible. In practice one constructs such spaces by finding a contractible space $E G$ with a good, free $G$ action. $B G$ is then the orbit space $E G / G$. For an easy example, consider the integers acting by translations on the real line. A model for the space $B \mathbb{Z}$ is then given by the circle $S^{1}=\mathbb{R} / \mathbb{Z}$.

The first homology group is always the abelianisation of $G$ :

$$
H_{1}(G)=G /[G, G] .
$$

Hopf also found a purely algebraic formula for the second homology group $H_{2}(G)$. Both these groups can generally be computed when one has a presentation of $G$ (indeed, often a subset of the relations suffices). Presentations for all the groups mentioned above are known. Nielsen and McCool had given a presentations of Aut $F_{n}$. Simplifications allowed Gersten $[18$ to compute its second homology group. For the mapping class group, the first presentations were found by $\mathrm{McCool}$ and via a more geometric approach by Thurston and Hatcher. Building on this, Harer [20] determined the second homology group. Further simplifications led to Wajnryb's convenient presentation in [45 and (with Birman) 3] with a set of generators as indicated in Figure 2. We summarize the results for the first two homology groups: for $n>4$ and $g>4$,

$$
\begin{aligned}
& H_{1}\left(\Sigma_{n}\right)=H_{2}\left(\Sigma_{n}\right)=\mathbb{Z} / 2 \mathbb{Z}, \\
& H_{1}\left(\operatorname{Aut} F_{n}\right)=H_{2}\left(\operatorname{Aut} F_{n}\right)=\mathbb{Z} / 2 \mathbb{Z}, \\
& H_{1}\left(\Gamma_{g, 1}\right)=0, \quad H_{2}\left(\Gamma_{g, 1}\right)=\mathbb{Z} .
\end{aligned}
$$

By the work of Culler and Vogtmann [7] and Harer [22] we know that both Out $F_{n}$ and $\Gamma_{g}$ have finite virtual cohomological dimensions:

$$
v c d\left(\mathrm{Out}_{n}\right)=2 n-3 \quad \text { and } \quad v c d\left(\Gamma_{g}\right)=4 g-5 .
$$

In particular this implies that the (co)homology in degrees above these dimensions is all torsion for both groups. Note that the virtual cohomological dimensions depend on $n$ and $g$. In contrast, in what follows we will only be interested in the (co)homology that is independent of $n$ and $g$.

2.3. Stable (co)homology and limit groups. The groups that we introduced in section 2.1 come in families $\left\{G_{n}\right\}_{n \geq 0}$ indexed by the natural numbers. For the symmetric groups $\Sigma_{n}$, the automorphisms of free groups Aut $F_{n}$ and the mapping class groups $\Gamma_{g, 1}$ there are canonical inclusions $G_{n} \hookrightarrow G_{n+1}$. Indeed, $\Sigma_{n} \hookrightarrow \Sigma_{n+1}$ identifies the smaller group with those permutations that leave the $(n+1)$-st letter fixed; Aut $F_{n} \hookrightarrow \operatorname{Aut} F_{n+1}$ with those automorphisms of $F_{n+1}$ that leave the $(n+1)$ st generator fixed and send the first $n$ generators to words not involving the $(n+1)$ st; and $\Gamma_{g, 1} \hookrightarrow \Gamma_{g+1,1}$ with those mapping classes that come from diffeomorphisms that restrict to the identity on $S_{g+1,1} \backslash S_{g, 1}$, which is a torus with two boundary components. In each case we define the limit groups as

$$
G_{\infty}:=\lim _{n \rightarrow \infty} G_{n} .
$$

It is natural to ask how the homology of $G_{n}$ is related to that of $G_{n+1}$ or $G_{\infty}$. In each of the three cases, the groups satisfy homology stability, which means that 
for a fixed degree the homology does not change once $n$ is large enough. This is the stable homology or equivalently the homology of the group $G_{\infty}$. The following maps in homology are isomorphisms for the degrees indicated:

$$
\begin{aligned}
H_{*}\left(\Sigma_{n}\right) \rightarrow H_{*}\left(\Sigma_{n+1}\right) & \text { for } *<(n+1) / 2, \\
H_{*}\left(\mathrm{Aut} F_{n}\right) \rightarrow H_{*}\left(\operatorname{Aut} F_{n+1}\right) & \text { for } *<(n-1) / 2, \\
H_{*}\left(\mathrm{Aut} F_{n}\right) \rightarrow H_{*}\left(\mathrm{Out} F_{n}\right) & \text { for } *<(n-3) / 2, \\
H_{*}\left(\Gamma_{g, 1}\right) \rightarrow H_{*}\left(\Gamma_{g+1,1}\right) & \text { for } *<2 g / 3, \\
H_{*}\left(\Gamma_{g, 1}\right) \rightarrow H_{*}\left(\Gamma_{g}\right) & \text { for } *<(2 g+1) / 3 .
\end{aligned}
$$

Homology stability theorems are generally quite tricky and difficult to prove. For the symmetric groups this was first studied by Nakaoka [38, for the mapping class groups by Harer [21] with improved ranges given by [31], 4], 40, and for the automorphism group of free groups by Hatcher and Vogtmann [26] (see also [27, [28], and [29]). For rational homology these stability ranges can often be improved. Indeed, for Aut $F_{n}$ this is $2 n / 3$ as used in the introduction; see [26].

The above results are crucial for us because it is the homology of the limit group $G_{\infty}$ that can be computed in each of the cases. And then, through homology stability, also some information on the homology of each $G_{n}$ can be obtained.

2.4. Products. At first it is counterintuitive that the homology of the larger group $G_{\infty}$ should be more easily determined than that of $G_{n}$. But note that we have the natural product maps

$$
\begin{aligned}
\Sigma_{n} \times \Sigma_{m} & \longrightarrow \Sigma_{n+m}, \\
\operatorname{Aut} F_{n} \times \operatorname{Aut} F_{m} & \longrightarrow \operatorname{Aut} F_{n+m}, \\
\Gamma_{g, 1} \times \Gamma_{h, 1} & \longrightarrow \Gamma_{g+h, 1} .
\end{aligned}
$$

The first two are given by having the first factor act on the first $n$ points or generators and the second factor on the last $m$. In the case of the mapping class group, the product map is induced by gluing $S_{g, 1}$ and $S_{h, 1}$ to the legs of a pairof-pants surface and extending the diffeomorphisms via the identity; see Figure 3 , Furthermore, the products are commutative up to conjugation by an element in the target group $G_{n+m}$. For the first two groups this is the element of the symmetric group $\Sigma_{n+m}$ that swaps the first $n$ with the last $m$ letters. On the other hand, for the mapping class group we need to conjugate by a twist of "legs" of the glued on the pair-of-pants surface, a braiding which is of infinite order. It is a standard fact that conjugation induces the identity on group homology. So we see that the products on homology are graded commutative.

On the level of spaces the above maps of groups induce a product on the disjoint union $M=\bigsqcup_{n} B G_{n}$ making it into a topological monoid. Monoids are not so well behaved, and we need to consider the associated group. Group completion is a powerful tool at the heart of algebraic $K$-theory, but also one of the more mysterious constructions. We devote the next section to it.

2.5. Group completion. The group completion $M \rightarrow \mathcal{G}(M)$ of a monoid $M$ is defined by the universal property that for any map of monoids $M \rightarrow G$ to a group $G$ there is a unique map of groups $\mathcal{G}(M) \rightarrow G$ making the obvious triangle commute. 


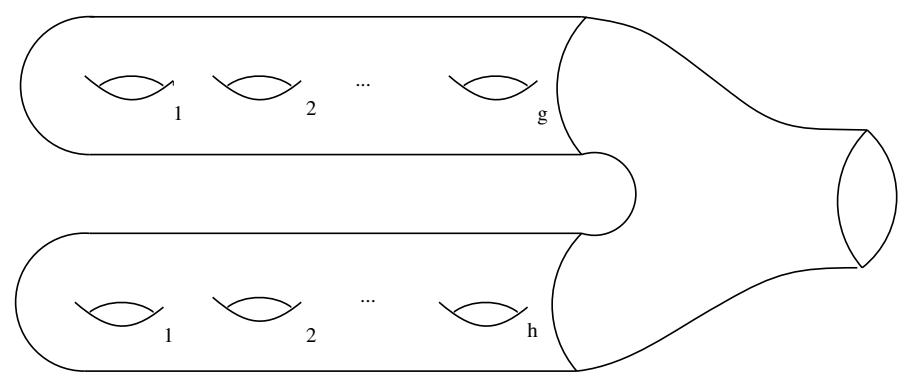

Figure 3. Pair-of-pants product for surfaces.

Thus for example, the group completion of the natural numbers are the integers $\mathbb{N} \rightarrow \mathbb{Z}$. More generally, when $M$ is abelian, its group completion is given by the set of equivalence classes of $M \times M$ under the relation: $\left(m_{1}, m_{2}\right) \sim\left(m_{1}^{\prime}, m_{2}^{\prime}\right)$ if and only if $m_{1} m_{2}^{\prime}=m_{1}^{\prime} m_{2}$. The map $M \rightarrow \mathcal{G}(M)$ is induced by the inclusion into the first factor $M \rightarrow M \times M$, though the map itself may not be injective. It is clear that when $M$ is already a group, its group completion is a canonical isomorphism $M \stackrel{\bar{S}}{\rightarrow}(M)$.

In order to have a well behaved construction in homotopy, algebraic notions most often have to be replaced by homotopy theoretic ones. In this setting the group completion functor $\mathcal{G}$ is replaced by the composite functor $\Omega B$ which associates to a topological monoid $M$ the space $\Omega B M$. Here $\Omega X=\operatorname{map}\left(S^{1}, X\right)$ denotes the loop spaces, the space of maps from a circle to $X$ that send a base point in $S^{1}$ to a base point in $X$. Every loop space has a product defined by the concatenation of loops which is associative up to homotopy and has inverses (given by reversing the direction of the loop) up to homotopy. When $M=G$ is a discrete group, $B G$ is the space mentioned before, and its loop space is well known to be homotopic to $G$ :

$$
\Omega B G \simeq \pi_{0}(\Omega B G) \simeq \pi_{1}(B G) \simeq G,
$$

and in the simplest case this gives $\Omega B \mathbb{Z} \simeq \Omega S^{1} \simeq \mathbb{Z}$.

In general, however, the homotopy of the monoid can be very different from that of its group completion. This can immediately be seen when looking at our examples from section 2.4. A connected component $B G_{n}$ of the monoid $M=$ $\bigsqcup_{n} B G_{n}$ has a non-trivial and non-commutative fundamental group $G_{n}$ and no higher homotopy. In contrast, every loop space has an abelian fundamental group (as $\pi_{1}(\Omega X)=\pi_{2}(X)$ ) and hence so does the group completion $\Omega B M$. Furthermore, as we will see, it has highly non-trivial higher homotopy groups. It is somewhat surprising despite this that the homology groups are related very nicely. The group completion theorem (first instances of which are proved in [2] and 39]) says that for suitable monoids $M$ the homology of a connected component $\Omega_{0} B M$ (they are all homotopic) of its group completion is the limit of the homology groups of its connected components. To come back to our three examples, we saw that $M=\bigsqcup_{n} B G_{n}$ has a product that is commutative on homology. This is a sufficient condition for the group completion theorem. We thus have

$$
H_{*}\left(G_{\infty}\right)=H_{*}\left(B G_{\infty}\right)=H_{*}\left(\Omega_{0} B\left(\bigsqcup_{n} B G_{n}\right)\right) .
$$


The interest in the above isomorphism comes from the fact that the group completion is a better behaved space than the monoid and that we may understand its homology more easily. For example, we see immediately that the limit group $G_{\infty}$ has the homology of a loop space. Indeed, a much stronger statement is true for our three examples. We will see that $G_{\infty}$ actually has the homology of an infinite loop space 1 (A space $X$ is an infinite loop space if there are spaces $X_{n}$ with $X=X_{0}$ and $\Omega X_{n+1}=X_{n}$ for all $n \geq 0$.) The fact that these spaces are infinite loop spaces puts some very strong restrictions on their homotopy type. Below we will see how to identify these infinite loop spaces. Indeed for the symmetric group, a classical theorem in homotopy theory, the Barratt-Priddy-Quillen theorem ([2] and [39]) asserts that the following two spaces are homotopy equivalent

$$
\Omega B\left(\bigsqcup_{n} B \Sigma_{n}\right) \simeq \Omega^{\infty} S^{\infty},
$$

where $\Omega^{\infty} S^{\infty}=\lim _{N \rightarrow \infty} \Omega^{N} S^{N}$ is the limit space of maps from $S^{N}$ to itself that fixes a chosen basepoint. Its homotopy groups are the notoriously hard to compute stable homotopy groups of spheres. However, for every prime $p$, the homology with $\mathbb{Z} / p \mathbb{Z}$ coefficients has been computed for $\Sigma_{\infty}$, and hence for $\Omega^{\infty} S^{\infty}$; see [1] and [9].

\section{Moduli sPaces AND their (CO)homology}

We switch from the algebraic to a more geometric point of view. The groups now have a natural topology and we need to distinguish between the homology of the group $G$ as a topological space and that of $B G$. So the group (co)homology of $G$ will always be thought of and written as the (co)homology of the space $B G$ which, as for discrete groups, is the quotient $E G / G$ of a contractible space $E G$, a good, free $G$ action.

3.1. Moduli spaces and characteristic classes. Our interest in groups and their (co)homology comes most often from an interpretation of the group $G$ as the automorphism group $\operatorname{Aut}(W)$ of some geometric object $W$. We like the model for $B \operatorname{Aut}(W)$ to be a (topological) moduli space in the sense that

(i) the points in $B \operatorname{Aut}(W)$ are representing objects isomorphic to $W$, and

(ii) any family of objects isomorphic to $W$ and indexed by a space $X$ corresponds to a continuous map $f: X \rightarrow B \operatorname{Aut}(W)$.

Such a family $E_{f}$ is called a $W$-bundle over $X$. The simplest example of such a bundle is the Cartesian product $W \times X$ which corresponds to the trivial map that sends every point in $X$ to the point in $B \operatorname{Aut}(W)$ that represents $W$ itself. If $f$ is the trivial map, then so is the map $f^{*}$ in cohomology. More generally, however, the elements in $f^{*}\left(H^{*}(B \operatorname{Aut}(W))\right) \subset H^{*}(X)$, which we call the characteristic classes of $E_{f}$, will give information about how twisted the family $E_{f} \rightarrow X$ is. It may be helpful to recall the well known theory of characteristic classes for vector bundles.

Example. To be concrete, take $W=\mathbb{C}^{n}$ a complex vector space of dimension $n$. Then $\operatorname{Aut}(W)=\mathrm{GL}(n, \mathbb{C})$ is the group of invertible, linear maps. A good model for $B \mathrm{GL}(n, \mathbb{C})$ is the complex Grassmannian manifold $\mathrm{Gr}^{\mathbb{C}}(n, \infty)$ of $n$-dimensional

\footnotetext{
${ }^{1}$ While for the first two groups this has long been known, for the mapping class group it came as a surprise; see [42. This is because the twisting for the mapping class groups, unlike for the symmetric groups or automorphism of free groups, does not square to the identity; it is only a braiding.
} 
$\mathbb{C}$-linear subspaces of $\mathbb{C}^{\infty}:=\bigcup_{n} \mathbb{C}^{n}$. This is a moduli space in the above sense. Its cohomology is well known to be

$$
H^{*}(B \mathrm{GL}(n, \mathbb{C}))=\mathbb{Z}\left[c_{1}, c_{2}, \ldots, c_{n}\right],
$$

where the $c_{i}$ are the universal Chern classes of degree $2 i$. For real vector spaces we have (the historically earlier) Pontryagin classes and Stiefel-Whitney classes. A standard reference for characteristic classes is [35].

These characteristic classes for vector bundles, which were discovered in the first half of the 20th century, have played a central role in the development of topology and geometry ever since. It is natural to ask,

What are the characteristic classes of bundles for more general $W$ ?

We are particularly interested when $W$ is a compact manifold and $\operatorname{Aut}(W)$ is its group of diffeomorphisms, or a finite simplicial complex and its group (up to homotopy) of homotopy equivalences. For example, when $W=S^{1}$ is a circle, we have homotopy equivalences

$$
\operatorname{Aut}(W)=\operatorname{Diff}^{+}\left(S^{1}\right) \simeq S^{1} \simeq \mathrm{GL}(1, \mathbb{C}),
$$

as every orientation preserving diffeomorphism is homotopic to a rotation which is in turn homotopic to $\mathrm{GL}(1, \mathbb{C})$. Thus the ring of characteristic classes for $S^{1}$ bundles is $H^{*}\left(B \operatorname{Diff}^{+}\left(S^{1}\right)\right)=\mathbb{Z}\left[c_{1}\right]$. With the proof of Mumford's conjecture and Galatius's theorem, we now also understand the characteristic classes, at least in the stable range, for $W$ an oriented surface and $W$ a simplicial complex of dimension one, as we will now explain.

3.2. Characteristic classes for surface bundles. We take $W$ to be an oriented, compact, smooth surface and its automorphism group to be the topological group Diff ${ }^{+}(W ; \partial)$ of orientation preserving diffeomorphisms (which fix the boundary pointwise, if it is not empty). To construct a topological moduli space, consider the space $\operatorname{Emb}\left(W, \mathbb{R}^{N}\right)$ of smooth embeddings of $W$ in Euclidean space, and let $\operatorname{Emb}\left(W, \mathbb{R}^{\infty}\right)=\bigcup_{N} \operatorname{Emb}\left(W, \mathbb{R}^{N}\right)$. One may think of this as the space of embedded and parameterized surfaces of type $W$ in $\mathbb{R}^{\infty}$. It is a consequence of Whitney's embedding theorem that this space is contractible. The diffeomorphism group $\operatorname{Diff}^{+}(W ; \partial)$ acts freely on it by precomposing an embedding by a diffeomorphism. The quotient space is the (topological) moduli space for $W$

$$
\mathcal{M}^{t o p}(W):=\operatorname{Emb}\left(W, \mathbb{R}^{\infty}\right) / \operatorname{Diff}^{+}(W ; \partial)=B \operatorname{Diff}^{+}(W ; \partial) .
$$

Its points are the unparameterized surfaces in $\mathbb{R}^{\infty}$ diffeomorphic to $W$. A point in it is a surface in $\mathbb{R}^{\infty}$. As we already mentioned, if $W=S_{g, 1}$ or $S_{g}$ and $g>1$ then the diffeomorphism group is homotopic to the mapping class group, and hence we have the homotopy equivalences

$$
\mathcal{M}^{t o p}\left(S_{g, 1}\right) \simeq B \Gamma_{g, 1} \quad \text { and } \quad \mathcal{M}^{t o p}\left(S_{g}\right) \simeq B \Gamma_{g} .
$$

3.2.1. Relation to moduli spaces of Riemann surfaces. The homology of the spaces above are also of particular interest to algebraic geometers because of their close relation to the moduli space $\mathcal{M}_{g}$ of Riemann surfaces of genus $g$. Indeed, there is an isomorphism of rational cohomologies

$$
H^{*}\left(\mathcal{M}_{g}\right) \otimes \mathbb{Q} \simeq H^{*}\left(\mathcal{M}_{g}^{t o p}\right) \otimes \mathbb{Q} \text {. }
$$


The basic reason for this is that $\mathcal{M}_{g}$ is the quotient of Teichmüller space, which is well known to be homeomorphic to $\mathbb{R}^{6 g-6}$ and thus contractible, by a good action of the mapping class group $\Gamma_{g}$. This action has only finite stabilizer groups as any Riemann surface can have at most finite automorphisms. As the rational cohomology of finite groups is trivial, a simple spectral sequence argument gives that $H^{*}\left(\mathcal{M}_{g}\right) \otimes \mathbb{Q} \simeq H^{*}\left(B \Gamma_{g}\right) \otimes \mathbb{Q}$, and hence the above isomorphism. Mumford [36] showed that $\mathcal{M}_{g}$ is a projective variety so that $\mathcal{M}_{g}$ is a moduli space for complex curves in the algebraic geometric setting. In the early 1980's Mumford [37. constructed characteristic classes $\kappa_{i}$ for the $\mathcal{M}_{g}$ and initiated the systematic study of its cohomology ring. These classes were also studied by Miller (and independently by Morita) in the topological setting who showed that

$$
H^{*}\left(B \Gamma_{\infty}\right) \otimes \mathbb{Q} \supset \mathbb{Q}\left[\kappa_{1}, \kappa_{2}, \ldots\right] .
$$

The proof of this result in [34] uses Harer's homology stability for the mapping class group and the commutativity of the product on the homology as described in section 2.4. In the light of this result, Mumford conjectured that the inclusion $(*)$ is indeed an isomorphism. This is now a theorem by Madsen and Weiss 33 . Indeed, they prove the following much stronger statement, first conjectured in 32 . It identifies the group completion with a certain infinite loop space.

Theorem [33. $\left.\Omega B\left(\bigsqcup_{g} B \Gamma_{g, 1} ; \partial\right)\right) \simeq \Omega^{\infty} \operatorname{MTSO}(2)$.

By the group completion theorem from section 2.5, we see that the space on the left-hand side has the cohomology of $\mathbb{Z} \times B \Gamma_{\infty}$. We will now define the space on the right-hand side and determine its rational cohomology.

3.2.2. Thom spaces and their rational cohomology. Let $\mathrm{Gr}^{+}(d, n)$ be the Grassmannian manifold of oriented $\mathbb{R}$-linear $d$ planes in $\mathbb{R}^{d+n}$. There are two canonical vector bundles over $\mathrm{Gr}^{+}(d, n)$ : the canonical $d$-bundle $\gamma_{d, n}$ with fibers over a plane $P \in \mathrm{Gr}^{+}(d, n)$ the vectors in $P$ and its orthogonal complement $\gamma_{d, n}^{\perp}$. We will only use $\gamma_{d, n}^{\perp}$ and its one-point compactification $\left(\gamma_{d, n}^{\perp}\right)^{c}$, also known as the Thom space of $\gamma_{d, n}^{\perp}$. There are embeddings $\operatorname{Gr}^{+}(d, n) \subset \mathrm{Gr}^{+}(d, n+1)$ induced by the inclusions $\mathbb{R}^{d+n} \subset \mathbb{R}^{d+n+1}$. The bundle $\gamma_{d, n+1}^{\perp}$, when restricted to this subset, is just $\gamma_{d, n}^{\perp} \oplus \mathbb{R}$, and on Thom spaces this gives an inclusion of the suspension $\Sigma\left(\gamma_{d, n}^{\perp}\right)^{+} \subset\left(\gamma_{d, n+1}^{\perp}\right)^{+}$. Using the suspension map $\Sigma: \operatorname{map}(Z, Y) \rightarrow \operatorname{map}(\Sigma Z, \Sigma Y)$, we can form the limit spact 2

$$
\Omega^{\infty} \operatorname{MTSO}(d):=\lim _{n \rightarrow \infty} \Omega^{d+n}\left(\gamma_{d, n}^{\perp}\right)^{c}
$$

here $\Omega^{k} X$ denotes the space of maps from $S^{k}$ to $X$ that take the point at infinity of $S^{k}=\left(\mathbb{R}^{k}\right)^{c}$ to the basepoint in $X$. The rational (co)homology of these spaces is well understood and can be computed by standard methods in algebraic topology; see for example [35, section 18]. For a connected component (they are all homotopic), we have

$$
H^{*}\left(\Omega_{0}^{\infty} \mathbf{M T S O}(d)\right) \otimes \mathbb{Q}=\Lambda\left(H^{>d}(B S O(d))[-d] \otimes \mathbb{Q}\right)
$$

\footnotetext{
${ }^{2}$ The notation MTSO reflects that the construction is very closely related to the construction of the classical oriented Thom spectrum MSO. Indeed, after a dimension shift (note that the Thom class in MTSO $(d)$ has dimension $-d)$, the spectra MTSO $(d)$ give a filtration of MSO. The $\mathbf{T}$ in the notation reminds us furthermore that the classifying maps of the tangent bundles are used in the construction and not those of the normal bundles as for MSO.
} 
here $\Lambda\left(V^{*}\right)$ for a graded vector space $V^{*}=\bigoplus_{n>0} V_{n}$ denotes the free graded commutative algebra on $V^{*}$. The $V^{*}$ in question here is given by $V^{n}=H^{d+n}(B S O(d)) \otimes$ $\mathbb{Q}$. As the rational classes of $H^{*}(B S O(d))$ are all of even degree, this is just a polynomial algebra. On taking $d=2$, Mumford's conjecture thus follows immediately from the above theorem.

Corollary. $H^{*}\left(B \Gamma_{\infty}\right) \otimes \mathbb{Q}=\mathbb{Q}\left[\kappa_{1}, \kappa_{2}, \ldots\right]$.

The theorem by Madsen and Weiss gives much more than just rational information. One is able to determine the divisibility of the $\kappa_{i}$ in the integral lattice $H^{*}\left(B \Gamma_{\infty}\right) /$ Tor. In [15] it was shown that the maximal divisor of $\kappa_{2 i}$ is 2 and that of $\kappa_{2 i-1}$ is the denominator of the $B_{i} / 2 i$ where $B_{i}$ is the $i$ th Bernoulli number. $\Omega^{\infty} \mathbf{M T S O}(2)$ also has a vast number of torsion classes; see 32 and [13. Indeed, for every even integer there is an infinite family of torsion homology classes (each essentially a copy of $\left.H_{*}\left(B \Sigma_{\infty}\right)\right)$. Only part of the first family had been detected previously; see [6.

3.3. Characteristic classes for graphs. In analogy to the moduli spaces of surfaces, Galatius [14 considers a moduli space $\mathcal{G}_{n}\left(\mathbb{R}^{\infty}\right)$ of embedded finite graphs in $\mathbb{R}^{\infty}$ that have fundamental group $F_{n}$ for a fixed $n$. Its topology is such that the collapse of a (non-loop) edge can be achieved by a continuous path 3 With this, $\mathcal{G}_{n}\left(\mathbb{R}^{\infty}\right)$ is in particular connected. Similarly, one can define a based version $\mathcal{G}_{n}\left(\mathbb{R}^{\infty} ; *\right)$ where each graph has a vertex at the origin. Using ideas from Igusa 30. and Culler and Vogtmann's outer space [7, Galatius establishes the weak homotopy equivalences

$$
\mathcal{G}_{n}\left(\mathbb{R}^{\infty} ; *\right) \simeq B \text { Aut } F_{n} \quad \text { and } \quad \mathcal{G}_{n}\left(\mathbb{R}^{\infty}\right) \simeq B \text { Out } F_{n}
$$

We will state now Galatius's theorem in analogue to Madsen and Weiss's theorem on the space level.

Theorem [14. $\Omega B\left(\bigsqcup_{n \geq 0} B\right.$ Aut $\left.F_{n}\right) \simeq \Omega^{\infty} S^{\infty}$.

In conjunction with the Barratt-Priddy-Quillen theorem (see section 2.4), we see that $\Sigma_{\infty} \hookrightarrow A u t F_{\infty}$ induces an isomorphism in homology. It is well known by a standard transfer argument that the homology (in positive degrees) of a finite group is all torsion; see [5]. Hence, as $\Sigma_{\infty}$ is a limit of finite groups, we have as an immediate consequence the analogue of Mumford's conjecture for Aut $F_{n}$.

Corollary. $H_{*}\left(B \operatorname{Aut} F_{\infty}\right) \otimes \mathbb{Q}=\mathbb{Q}$ is concentrated in degree 0 .

Previous evidence for Galatius's theorem came in the form of a remark by Hatcher in 24, who proved that $\Omega^{\infty} S^{\infty}$ is a direct factor of the left-hand side. Hatcher already raised the question whether it could be an equivalence and in particular whether the rational homology of $A u t F_{\infty}$ is trivial. Hatcher and Vogtmann in 25] proved that $H_{*}\left(\operatorname{Aut} F_{n}\right) \otimes \mathbb{Q}=0$ for $0<*<7$ with the exception of $H_{4}\left(\right.$ Aut $\left.F_{4}\right) \otimes \mathbb{Q}=\mathbb{Q}$, providing strong evidence for the homological conjecture. Further evidence for the conjecture on the space level was given by a theorem by Igusa [30]. It says that the linearisation map $L: \operatorname{Aut} F_{n} \rightarrow \operatorname{GL}(n, \mathbb{Z})$ (see section

\footnotetext{
${ }^{3}$ The topology is somewhat delicate. In particular, one wants the graph to be embedded in such a way that every point in $\mathbb{R}^{\infty}$ has a neighborhood which either does not intersect the graph, or intersects it in a small interval (part of an edge), or contains a neighborhood of a vertex (and no more). Collapsing an edge has to be done in such a way that this is always satisfied.
} 


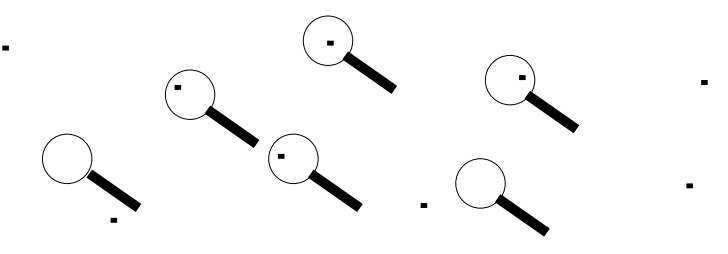

Figure 4. A configuration of points in $\mathbb{R}^{N}$ sampled by a magnifying glass.

2.1.2) on classifying spaces and after group completion (i.e., application of $\Omega B$ ) factors through $\Omega^{\infty} S^{\infty}$.

\section{TOWARDS A PROOF}

Our very rough sketch here simultaneously treats the Barratt-Priddy-Quillen theorem, the Madsen-Weiss theorem, as well as Galatius's theorem. We mainly follow [14] (and in parts the conceptually closely related [16]). In particular, we emphasize the role of the scanning map.

4.1. Scanning. In abstract terms, the scanning map can be applied to topological moduli spaces where a point is an embedded object $W$ in $\mathbb{R}^{N}$, for $N$ large. The idea is that the scanning map at the point $W \subset \mathbb{R}^{N}$ records the local, microscopic picture as a magnifying glass sweeps through $\mathbb{R}^{N}$, thus defining a map from $\mathbb{R}^{N}$ to the space of all such local pictures. If the topological moduli space contains only compact objects, then the scanning map will record always the empty set near infinity and thus can be extended to the sphere $S^{N}=\left(\mathbb{R}^{N}\right)^{c}$.

We first consider the simplest and most well known case when $W$ is a set of $n$ points. The ideas here go back to Segal and McDuff. The associated moduli space is the configuration space $\mathcal{C}_{n}\left(\mathbb{R}^{N}\right)$ of $n$ distinct, unordered points in $\mathbb{R}^{N}$. When scanning, the lens of the magnifying glass can be taken small enough such that it only sees at most one point. Identifying the lens with a ball $B^{N}$ in $\mathbb{R}^{N}$, we see that the space of all local pictures is just the sphere $S^{N}=\left(B^{N}\right)^{c}$ where the point at infinity corresponds to the lens giving us the view of the empty set. As the lens moves across $\mathbb{R}^{N}$ for a given configuration, we thus get a map from $\mathbb{R}^{N}$ to $S^{N}$. But away from a compact set containing the $n$ points, the lens sees nothing and the map is constant. We may therefore extend the map to $S^{N}=\left(\mathbb{R}^{N}\right)^{c}$ by sending the point at infinity to a point representing the empty set. Thus scanning defines a map

$$
\bigsqcup_{n} \mathcal{C}_{n}\left(\mathbb{R}^{N}\right) \longrightarrow \Omega^{N} S^{N}
$$

Similarly, scanning can be applied to the moduli space

$$
\mathcal{M}^{\text {top }}\left(S_{g}\right)^{N}:=\operatorname{Emb}\left(S_{g}, \mathbb{R}^{N}\right) / \operatorname{Diff}^{+}\left(F_{g}\right)
$$

of surfaces of type $S_{g}$ embedded in $\mathbb{R}^{N}$. This time, unless the lens sees nothing, it will see aproximately an oriented 2-plane intersecting $B^{N}$, which is the tangent plane $T_{x}$ of the nearest point $x$ on the surface to the center of the lens. Identifying $B^{N}$ with $\mathbb{R}^{N}$, this defines a two-dimensional subspace $T_{x}-x$ of $\mathbb{R}^{N}$ and a vector $x$ perpendicular to it. Thus we see that the Thom space $\left(\gamma_{2, N-2}^{\perp}\right)^{c}$ is the space of all local data with the point at infinity corresponding again to the empty lens. Thus 


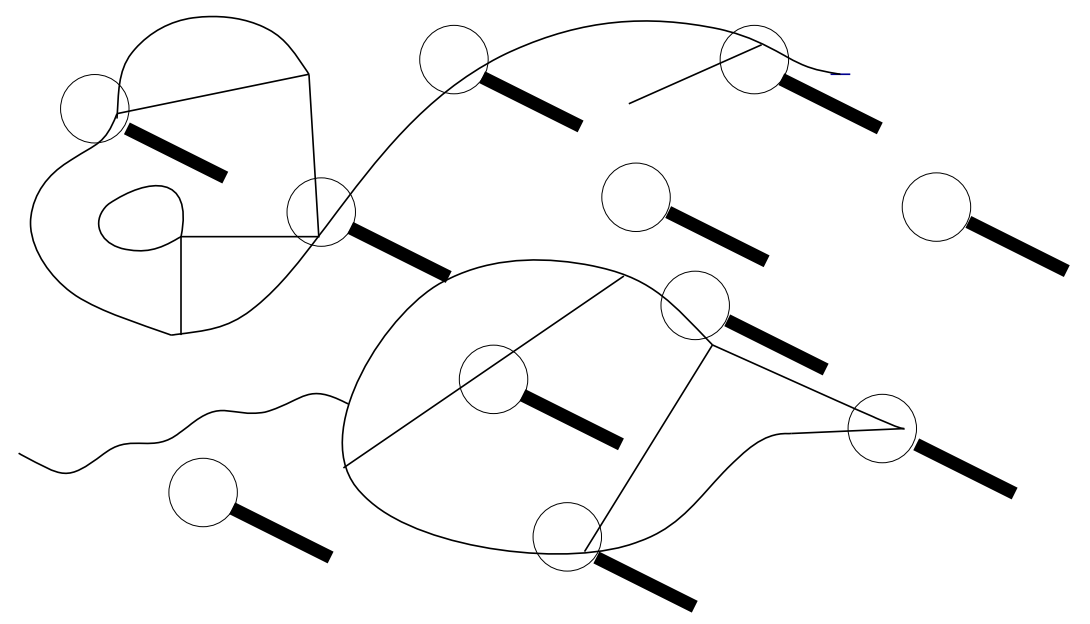

FiguRE 5. A graph in $\mathbb{R}^{N}$ sampled by a magnifying glass.

for every point in $\mathbb{R}^{N}$, we get a point in $\left(\gamma_{2, N-2}^{\perp}\right)^{c}$, and again we can extend this map continuously to the compactification $S^{N}=\left(\mathbb{R}^{N}\right)^{c}$. Thus scanning defines a map

$$
\bigsqcup_{g} \mathcal{M}^{t o p}\left(S_{g}\right)^{N} \longrightarrow \Omega^{N}\left(\gamma_{2, N-2}^{\perp}\right)^{c}
$$

The case of graphs is similar, only that the space of all local data is much harder to identify. Indeed, Galatius spends considerable effort to show that a map from the $N$-sphere $S^{N}$ to the space of local data in dimension $N$ induces an isomorphism on homotopy groups in degrees $2 N-c$ for some fixed $c$ independent of $N$. As $N$ goes to infinity this shows that the space of local data is indeed weakly homotopic to $\Omega^{\infty} S^{\infty}$, and scanning defines a map

$$
\bigsqcup_{n} \mathcal{G}_{n}\left(\mathbb{R}^{\infty}\right) \rightarrow \Omega^{\infty} S^{\infty}
$$

4.2. Spaces of manifolds and graphs. As with the group completion map, the above maps can of course not be homotopy equivalences. Not all path components in the target are hit and the maps are not injective on fundamental groups. We need to enlarge our moduli spaces. Instead of considering only compact objects in $\mathbb{R}^{N}$, we consider manifolds and graphs in $\mathbb{R}^{N}$ that may also be non-compact. So let $\Phi^{N, N}$ be the space of all manifolds $W$ of a given dimension $d$ (or graphs $G$ ) in $\mathbb{R}^{N}$. Each $W$ (respectively $G$ ) has to be a closed subset of $\mathbb{R}^{N}$ but may extend to infinity. It also need not be connected. $\Phi^{N, N}$ is topologized in such a way that manifolds or graphs can be pushed continuously to infinity. To get a feeling for this topology, note that expanding radially away from a point not in $W$ (or $G$ ) defines a path from any point in $\Phi^{N, N}$ to the empty set, which we take to be the basepoint. Thus in particular, $\Phi^{N, N}$ is path connected. We have a filtration

$$
\Phi^{N, N} \supset \cdots \supset \Phi^{N, 1} \supset \Phi^{N, 0},
$$

where $\Phi^{N, i}$ contains only those $W($ or $G)$ that are subsets of $\mathbb{R}^{i} \times(0,1)^{N-i}$. In particular, they are compact in the last $N-i$ coordinate directions, and the last 
one is homotopy equivalent to our moduli spaces

$$
\Phi^{N, 0} \simeq \bigsqcup_{n} \mathcal{C}\left({ }_{n}\left(\mathbb{R}^{N}\right), \quad \bigsqcup_{g} \mathcal{M}^{\text {top }}\left(S_{g}\right)^{N}, \quad \text { or } \bigsqcup_{n} \mathcal{G}_{n}\left(\mathbb{R}^{N}\right) .\right.
$$

To prove the theorems by Barratt-Priddy-Quillen, Madsen-Weiss or Galatius, one would like to complete three steps. For configuration spaces this is an argument that essentially goes back to Segal [41].

Step 1. $\Phi^{N, N}$ is homotopic to the space of local data of the scanning map.

This is relatively easy. The key here is that the topology of $\Phi^{N, N}$ allows us to push radially away from the origin. At the end of the homotopy, what is left is the local data (at the origin).

Step 2. $\Phi^{N, k} \rightarrow \Omega \Phi^{N, k+1}$ is a homotopy equivalence for $k>04$

We can construct a map as follows. For each $t$ one can define a map $\Phi^{N, k} \rightarrow$ $\Phi^{N, k+1}$ by sending $W$ to its translate $W-t e_{k+1}$ by $t$ in the $(k+1)$ st direction. So as $t$ goes to infinity, $W$ gets pushed out of sight and we can extend the map to $S^{1}=\mathbb{R}^{c}$ by sending the point at infinity to the empty set. The case for graphs is just the same.

In the case of configuration spaces, it is straight forward to prove that this is a homotopy equivalence. But some extra argument is required in the case of higher dimensional manifolds and graphs.

Step 3. $\lim _{N \rightarrow \infty} \Phi^{N, 1}$ is homotopic to the classifying space of $\bigsqcup_{n} B$ Aut $W_{n}$.

Just as Step 2, this is straightforward for configuration spaces. More generally, it is not difficult to see that in the manifold case $\lim _{N \rightarrow \infty} \Phi^{N, 1}$ is the classifying space of the $d$-dimensional cobordism category as studied in topological field theory; see for example [16. Similarly, in the graph case, one sees that $\lim _{N \rightarrow \infty} \Phi^{N, 1}$ is the classifying space of a cobordism category of graphs.

Though interesting in themselves, these identifications of $\lim _{N \rightarrow \infty} \Phi^{N, 1}$ do not yet allow one to make deductions for $B$ Aut $W_{\infty}$ or $B$ Aut $W_{n}$. In order to do so, one wants to apply a group completion theorem following the arguments in [42. Two things are needed in order to apply it. First one needs to show that the classifying space of the cobordism category is homotopic to that of its subcategory in which the cobordisms are such that each component has a non-empty out-going boundary. This is done in 16 for manifolds (of all dimensions $\geq 2$ ) and in 14 for graphs. Secondly, one needs homology stability - which of course we have for graphs and surfaces.

We note here that in [17] a proof of Madsen and Weiss's theorem in the form stated above is given that no longer uses homology stability. Indeed, Galatius and Randal-Williams show that the inclusion of the monoid $\bigsqcup_{n} B$ Aut $W_{n}$ into the whole category induces a homotopy equivalence on classifying spaces after group completion (i.e., applying $\Omega B$ ).

\footnotetext{
${ }^{4}$ This statement is equivalent to saying that $\Phi^{N, k+1}$ is homotopic to $B \Phi^{N, k}$ and the connected components of $\Phi^{N, k}$ form a group. The product in $\Phi^{N, k}$ can be defined as follows: Given two graphs $W_{1}$ and $W_{2}$, move $W_{2}$ to its translate $W_{2}+e_{k+1}$. The resulting manifolds are disjoint and one can take the disjoint union. Using a homotopy $[0,2] \simeq[0,1]$, one can move the manifold back into $\Phi^{N, k}$. Showing that $\Phi^{N, k+1} \simeq B \Phi^{N, k}$ is not too difficult. Showing that the connected components form a group requires some argument. Note that it is here that we require the condition on $k$ as the connected components certainly do not form a group when $k=0$.
} 
TABLE 1

\begin{tabular}{|c|c|c|c|}
\hline$G_{n}$ & $*<$ stab range & $\operatorname{Aut} W_{n}$ & $\Omega B\left(\bigsqcup_{n} B \operatorname{Aut} W_{n}\right)$ \\
\hline$\Sigma_{n}$ & $(n+1) / 2$ & $\operatorname{Diff}(n$ pts $)$ & $\Omega^{\infty} S^{\infty}$ \\
\hline$\Gamma_{g, 1}$ & $(2 g) / 3$ & $\operatorname{Diff}^{+}\left(S_{g, 1} ; \partial\right)$ & $\Omega^{\infty} \operatorname{MTSO}(2)$ \\
\hline $\mathcal{N}_{g, 1}$ & $(g-3) / 3$ & $\operatorname{Diff}\left(N_{g, 1} ; \partial\right)$ & $\Omega^{\infty} \operatorname{MTO}(2)$ \\
\hline . & $\cdot$ & $\operatorname{Diff}^{+}\left(T_{g, 1}^{2 k} ; \partial\right)$ & $\Omega^{\infty} \mathbf{M T S O}(2 k)^{\langle k\rangle}$ \\
\hline Aut $F_{n}$ & $(n-1) / 2$ & $\operatorname{HtEq}\left(G_{n} ; *\right)$ & $\Omega^{\infty} S^{\infty}$ \\
\hline $\mathcal{H}_{g, 1}$ & $(g-1) / 2$ & $\operatorname{Diff}^{+}\left(H_{g} ; D^{2} \subset \partial\right)$ & $\Omega^{\infty} S^{\infty} B S O(3)_{+}$ \\
\hline . & & $\operatorname{Diff}^{+}\left(L_{g, 1} ; \partial\right)$ & $\Omega^{\infty} S^{\infty} B S O(4)_{+}$ \\
\hline
\end{tabular}

\section{Survey of Further Results}

Madsen and Weiss's theorem as well as Galatius's theorem have been generalized in several directions. It is convenient to summarize some of these results in Table 1 .

The first column of Table 1 gives the discrete group $G_{n}$ to be considered; the second column lists the integer $k$ so that the map $G_{n} \rightarrow G_{n+1}$ induces a homology isomorphisms in degrees less than $k$; the third column gives the automorphism group Aut $W_{n}$ of the underlying geometric object; and finally, the fourth column contains a space weakly homotopy equivalent to the group completion $\Omega B\left(\bigsqcup_{n \geq 0} B A u t W_{n}\right)$ and with the same homology as $\mathbb{Z} \times B$ Aut $W_{\infty}$.

We now briefly discuss the new entries. The first is a consequence of Madsen and Weiss's work once homology stability has been established. The latter are extensions that use and develop the methods developed in [14].

5.1. Non-orientable surfaces. Let $N_{g, 1}$ be a non-orientable surface of genus $g$ (i.e., a connected sum of $g$ copies of $\mathbb{R} P^{2}$ 's) with a boundary component. Its diffeomorphisms group $\operatorname{Diff}\left(N_{g, 1} ; \partial\right)$ has the same homotopy type as the associated mapping class group $\mathcal{N}_{g}$ by [8. As for oriented surfaces, extending diffeomorphisms by the identity map over a glued-on pair-of-pants surface induces a product $\mathcal{N}_{g, 1} \times \mathcal{N}_{h, 1} \rightarrow \mathcal{N}_{g+h, 1}$. Wahl [44] showed that $\mathcal{N}_{g, 1}$ satisfies homology stability, and Randal-Williams [40] improved Wahl's stability range to $(n-3) / 3$. (In this paper Randal-Williams also proves homology stability for surfaces with more exotic tangential structures (such as framed, spin and pin) which we have not listed in the above table.) The space $\Omega^{\infty} \mathbf{M T O}(2)$ is constructed just as in the oriented case only that the Grassmannian of non-oriented planes is considered. This gives the stable homology of the non-oriented mapping class group as

$$
H_{*}\left(\mathcal{N}_{\infty}\right) \otimes \mathbb{Q}=\mathbb{Q}\left[\xi_{i}\right] \quad \text { with } \operatorname{deg} \xi_{i}=4 i .
$$

5.2. A $(k-1)$-connected $2 k$-manifold. The connected sum of $g$ copies of $S^{k} \times S^{k}$ is a higher dimensional analogue of a surface $S_{g}=\#{ }_{g} S^{1} \times S^{1}$. We remove an open ball $B^{2 k}$, and let

$$
T_{g, 1}^{2 k}:=\left(\#_{g} S^{k} \times S^{k}\right) \backslash B^{2 k} .
$$


Consider diffeomorphisms that fix the boundary pointwise. Then a product can be constructed by gluing as for surfaces. At the moment we do not know whether the classifying spaces of the diffeomorphism groups satisfy homology stability. Nevertheless, generalizing their arguments in [17, for $k>2$ Galatius and RandalWilliams have identified the group completion of the associated monoid with a slight modification of the space $\Omega^{\infty} \operatorname{MTSO}(2 k)$. To define this, consider the $k$ connected cover $\pi: \operatorname{Gr}^{+}(2 k, n)\langle k\rangle \rightarrow \operatorname{Gr}^{+}(2 k, n)$ and the pullback bundle $\pi^{*}\left(\gamma_{2 k, n}^{\perp}\right)$ of the universal bundle $\gamma_{2 k, n}^{\perp}$. (For a space $X$, its 1 -connected cover $X\langle 1\rangle \rightarrow X$ is just its universal cover. The $k$-connected cover $X\langle k\rangle \rightarrow X$ is a generalization of this in that $X\langle k\rangle$ has trivial homotopy groups for $* \leq k$ and the same homotopy groups as $X$ for $*>k$.) The space we are looking for is

$$
\Omega^{\infty} \operatorname{MTSO}(2 k)^{\langle k\rangle}:=\lim _{n \rightarrow \infty} \Omega^{2 k+n}\left(\pi^{*}\left(\gamma_{2 k, n}^{\perp}\right)\right)^{c} .
$$

5.3. Handlebody in dimension 3. Diffeomorphism of the three-dimensional handlebody $H_{g}:=\#_{g} S^{1} \times D^{2}$ of genus $g$ restricts to diffeomorphisms of the boundary surface. Furthermore, its connected components are contractible. This is still the case when all diffeomorphisms fix a disk $D^{2} \subset \partial$ on the boundary. (This fixed disk is used to define a product.) Its mapping class group $\mathcal{H}_{g, 1}$ may be identified with a subgroup of $\Gamma_{g, 1}$. Hatcher and Wahl 29] proved that these groups satisfy homology stability. By considering the handlebody as a thickened graph and adopting Galatius's proof from [14, Hatcher showed recently that the group completion in this case is

$$
\Omega^{\infty} S^{\infty} B S O(3)_{+}=\lim _{k \rightarrow \infty} \Omega^{k} S^{k}\left(B S O(3)_{+}\right) .
$$

The proof uses another ingredient, the Smale conjecture (see [23]), which states that $\operatorname{Diff}\left(D^{3}\right) \simeq \mathrm{O}(3)$. Here $X_{+}$denotes $X$ with a disjoint basepoint. In particular, this gives the stable homology of the handlebody mapping class group as

$$
H_{*}\left(\mathcal{H}_{\infty}\right) \otimes \mathbb{Q}=\mathbb{Q}\left[\kappa_{2 i}\right] \quad \text { with } \operatorname{deg} \kappa_{2 i}=4 i,
$$

and where $\kappa_{2 i}$ is the Mumford-Morita-Miller class restricted to the handlebody mapping class group; see also [19].

5.4. A simple three-dimensional manifold. Finally, the bottom line is also work by Hatcher, recently announced. It concerns the three-dimensional manifold

$$
L_{g, 1}:=\left(\#_{g} S^{1} \times S^{2}\right) \backslash B^{3},
$$

the connected sum of $n$ copies of $S^{1} \times S^{2}$ with an open ball removed so that a product can be defined. Again Hatcher uses a modification of Galatius's argument for graphs 14 and the Smale conjecture. Note however, that in this case the connected components of the diffeomorphism groups are not contractible and we do not know whether the classifying spaces of these groups satisfy homology stability.

\section{Conclusion And Future Directions}

We have seen that the method of scanning can be applied to topological moduli spaces $\mathcal{M}^{\text {top }}(W)$ of objects isomorphic to $W$ embedded in $\mathbb{R}^{\infty}$. The target $T$ of the scanning map is a highly structured space, an infinite loop space. In the case of zero-dimensional manifolds, graphs, and two-dimensional manifolds the target of the scanning map $T$, in the presence of homology stability, gives a better and 
better approximation to the homology of the moduli space $\mathcal{M}^{t o p}(W)$ as the complexity of $W$ grows. In the previous section we encountered manifolds $W$ of higher dimensions for which the scanning map induces a homotopy equivalence from the group completion of the associated monoid to the target $T$ but for which we do not (yet) have homology stability.

One is naturally led to ask the following questions: Can Table 1 be completed and homology stability results be found for certain types of manifolds? Are there any other families of manifolds that can be added to Table 1? Indeed, are there other geometric objects to which the scanning method can be applied? Galatius considered finite one-dimensional complexes. Can the methods be pushed to higherdimensional finite complexes?

We emphasized the point of view of moduli spaces and characteristic classes for manifold bundles. For for every oriented, closed, $d$-dimensional manifold $W$, scanning gives a map $\alpha: \mathcal{M}^{\text {top }}(W) \rightarrow \Omega^{\infty} \mathbf{M T S O}(d)$. So the cohomology of $\Omega^{\infty} \mathbf{M T S O}(d)$ provides characteristic classes for all oriented $d$-manifolds simultaneously. Ebert has shown that for $d$ even every rational cohomology class $c \in$ $H^{*}\left(\Omega^{\infty} \mathbf{M T S O}(d)\right) \otimes \mathbb{Q}$ is detected by some manifold, i.e., $\alpha^{*}(c)$ is non-zero for some $W$. But this fails for $d$ odd; see [10] and 11. This suggests that the scanning map for $d$ odd is not optimal and should factor through a space $X(d)$. For $d=1$, this space is $X(1)=\Omega^{\infty} S^{\infty} B S O(2)_{+}$. Hatcher's last example suggests a similar solution for $d=3$.

We have tried to give here a glimpse into an active area of research that uses new techniques to study basic questions in geometry and topology. Galatius's work has been central to this development.

\section{ACKNOWLEDGMENTS}

The author is grateful to Søren Galatius, Allen Hatcher, and Oscar RandalWilliams for their comments on an earlier draft of this paper.

\section{ABOUT THE AUTHOR}

The author was a student of Ralph Cohen at Stanford University and a postdoc of Graeme Segal at Cambridge University. Since then she has been working at Oxford University. Since 2007 she has been one of the founding editors of the Journal of Topology. She was elected a Fellow of the Royal Society in 2008.

\section{REFERENCES}

[1] S. Araki, T. Kudo, Topology of $H_{n}$-spaces and $H_{n}$-squaring operations, Mem. Fac. Sci. Kyushu Univ. Ser A 10 (1956), 85-120. MR0087948 (19:442b)

[2] M. Barratt, S. Priddy, On the homology of non-connected monoids and their associated groups, Comment. Math. Helv. 47 (1972), 1-14. MR0314940(47:3489)

[3] J. Birman, B. Wajnryb, Presentations of the mapping class group. Errata, Israel J. Math. 88 (1994), no. 1-3, 425-427. MR.1303506 (95j:57014)

[4] S. K. Boldsen, Improved homological stability for the mapping class group with integral or twisted coefficients, Mat. Zeit., to appear arXiv:0904.3269

[5] K. S. Brown, Cohomology of groups, Graduate Texts in Mathematics 87 Springer-Verlag (1982). MR672956 (83k:20002)

[6] R. Charney, R. Lee, An application of homotopy theory to mapping class groups, J. Pure Appl. Algebra 44 (1987), no. 1-3, 127-135. MR885100(88m:55024)

[7] M. Culler, K. Vogtmann, Moduli of graphs and automorphisms of free groups, Invent. Math. 84 (1986), no. 1, 91-119. MR830040 (87f:20048) 
[8] C. J. Earle and J. Eells, A fibre bundle description of Teichmüller theory, J. Differential Geometry 3 (1969), 19-43. MR0276999(43:2737a)

[9] E. Dyer, R. Lashof, Homology of iterated loop spaces., Amer. J. Math. 84 (1962), 35-88. MR0141112 (25:4523)

[10] J. Ebert, A vanishing theorem for characteristic classes of odd-dimensional manifold bundles, arXiv:0902.4719

[11] J. Ebert, Algebraic independence of generalized Morita-Miller-Mumford classes, Algebr. Geom. Topol. 11 (2011), 69-105. MR.2764037

[12] B. Farb, D. Margalit, A primer on mapping class groups, Princeton University Press, to appear.

[13] S. Galatius, Mod p homology of the stable mapping class group, Topology 43 (2004), no. 5, 1105-1132. MR2079997 (2006a:57020)

[14] S. Galatius, Stable homology of automorphism groups of free groups, Ann. of Math. 173 (2011), 705-768. math/0610216 MR2784914

[15] S. Galatius, I. Madsen, U. Tillmann, Divisibility of the stable Miller-Morita-Mumford classes, J. Amer. Math. Soc. 19 (2006), no. 4, 759-779. MR.2219303 (2006m:57039)

[16] S. Galatius, I. Madsen, U. Tillmann, and M. Weiss, The homotopy type of the cobordism category, Acta Math. 202 (2009), no. 2, 195-239. MR.2506750 (2011c:55022)

[17] S. Galatuis and O. Randal-Williams, Monoids of moduli spaces of manifolds, Geom. Topol. 14 (2010), no. 3, 1243-1302. MR2653727 (2011j:57047)

[18] S. M. Gersten, A presentation for the special automorphism group of a free group, J. Pure Appl. Algebra 33 (1984), no. 3, 269-279. MR761633 (86f:20041)

[19] J. Giansiracusa, U. Tillmann, Vanishing of universal charcteristic classes for handlebody groups and boundary bundles, arXiv:0910.5367

[20] J. Harer, The second homology of the mapping class group of an orientable surface, Invent. Math. 72 (1983), 221-239. MR700769 (84g:57006)

[21] J. L. Harer, Stability of the homology of the mapping class groups of orientable surfaces, Ann. of Math. (2) 121 (1985), no. 2, 215-249. MR786348 (87f:57009)

[22] J. L. Harer, The virtual cohomological dimension of the mapping class group of an orientable surface, Invent. Math. 84 (1986), no. 1, 157-176. MR830043(87c:32030)

[23] A. Hatcher, A proof of the Smale conjecture, Diff $\left(S^{3}\right) \simeq S O(3)$, Ann. of Math. 117 (1983), 553-607. MR701256 (85c:57008)

[24] A. Hatcher, Homological stability for automorphism groups of free groups, Comment. Math. Helv. 70 (1995), no. 1, 39-62. MR.1314940 (95k:20030)

[25] A. Hatcher, K. Vogtmann, Rational homology of Aut $\left(F_{n}\right)$, Math. Res. Lett. 5 (1998), no. 6, 759-780. MR1671188 (99m:20127)

[26] A. Hatcher, K. Vogtmann, Cerf theory for graphs, J. London Math. Soc. 58 (1998), 633-655. MR.1678155 (2000e:20041)

[27] A. Hatcher, K. Vogtmann, Homology stability for outer automorphism groups of free groups, Algebr. Geom. Topol. 4 (2004), 1253-1272. MR2113904 (2005j:20038)

[28] A. Hatcher, K. Vogtmann, N. Wahl, Erratum to: Homology stability for outer automorphism groups of free groups, Algebr. Geom. Topol. 6 (2006), 573-579. MR2220689 (2006k:20069)

[29] A. Hatcher, N. Wahl, Stabilization for mapping class groups of 3-manifolds, Duke Math. J. 155 (2010), 205-269. MR2736166

[30] K. Igusa, Higher Franz-Reidemeister torsion. AMS/IP Studies in Advanced Mathematics, 31. American Mathematical Society (2002). MR:1945530 (2004f:19003)

[31] N. V. Ivanov, Stabilization of the homology of Teichmüller modular groups, (Russian) Algebra i Analiz 1 (1989), no. 3, 110-126; translation in Leningrad Math. J. 1 (1990), no. 3, 675-691. MR:1015128 (91g:57010)

[32] I. Madsen and U. Tillmann, The stable mapping class group and $Q\left(C P_{+}^{\infty}\right)$, Invent. Math. 145 (2001), no. 3, 509-544. MR1856399 (2002h:55011)

[33] I. Madsen and M. Weiss, The stable moduli space of Riemann surfaces: Mumford's conjecture, Ann. of Math. 165 (2007), 843-941. MR2335797(2009b:14051)

[34] E. Y. Miller, The homology of the mapping class group, J. Differential Geom. 24 (1986), no. 1, 1-14. MR857372 (88b:32051)

[35] J. W. Milnor and J. D. Stasheff, Characteristic classes; Ann. of Math. Stud., 76 Princeton University Press, (1974). MR0440554(55:13428) 
[36] D. Mumford, Geometric Invariant Theory, Ergebnisse der Mathematik 34, Springer (1965) (Third enlarged edition with J. Fogarty, F. Kirwan (1994)). MR0214602 (35:5451)

[37] D. Mumford, Towards an enumerative geometry of the moduli space of curves, Arithmetic and geometry, Vol. II, 271-328, Progr. Math., 36, Birkhäuser Boston, Boston, MA, 1983. MR.717614 (85j:14046)

[38] M. Nakaoka, Decomposition theorem for homology groups of symmetric groups, Ann. of Math. 271 (1960) 16-42. MR0112134 (22:2989)

[39] E. Friedlander, B. Mazur, Filtrations on the homology of algebraic varieties. With an appendix by Daniel Quillen. Mem. Amer. Math. Soc. 110 (1994). MR.1211371 (95a:14023)

[40] O. Randal-Williams, Resolutions of moduli spaces and homological stability, arXiv:0909.4278

[41] G. Segal, Configuration-spaces and iterated loop-spaces, Invent. Math. 21 (1973), 213-221. MR0331377 (48:9710)

[42] U. Tillmann, On the homotopy of the stable mapping class group, Invent. Math. 130 (1997), no. 2, 257-275. MR 1474157 (99k:57036)

[43] K. Vogtmann, The cohomology of automorphism groups of free groups, International Congress of Mathematicians. Vol. II, Eur. Math. Soc., Zürich (2006), 1101-1117. MR2275637 (2007k:20090)

[44] N. Wahl, Homological stability for the mapping class groups of non-orientable surfaces, Invent. Math. 171 (2008), no. 2, 389-424. MR2367024 (2008m:57047)

[45] B. Wajnryb, A simple presentation for the mapping class group of an orientable surface, Israel J. Math. 45 (1983), no. 2-3, 157-174. MR719117 (85g:57007)

Mathematical Institute, Oxford University, 24-29 St Giles St., Oxford OX1 3LB, UNITED KINGDOM

E-mail address: tillmann@maths.ox.ac.uk 